\title{
Eigenvalues and Three-Term Approximation of Fourier Series Solution of Heat Conduction Transients, Valid for $0.02<\mathbf{F o}<\infty$ and All Bi
}

\author{
A. Ostrogorsky ${ }^{1}$ \\ ${ }^{1}$ Illinois Institute of Technology \\ e-mail: AOstrogo@iit.edu
}

\begin{abstract}
For transient conduction/diffusion driven by convection boundary conditions, in plates, cylinders and spheres, a set of correlations is presented providing explicit one-, two- and threeterm approximations of Fourier series solution. The correlations yield eigenvalues $\left(\lambda_{1}, \lambda_{2}\right.$ and $\left.\lambda_{3}\right)$ and coefficients $\left(A_{1}, A_{2}\right.$ and $\left.A_{3}\right)$ with less than $0.6 \%$ error. The correlations are more precise than tables or charts available in textbooks, and do not require interpolation between given values or curves.
\end{abstract}

Keywords: Eigenvalues, heat conduction, diffusion, Fourier series.

\section{Introduction}

Transient heat conduction problems in finite solids with convective boundary conditions are common. While exact analytical solutions are available, their precision is limited by the $\pm 10 \%$ uncertainty in the convection heat transfer coefficient $h$ and Biot number,

$$
B i=\frac{h L}{k}
$$

where $\mathrm{k}$ is thermal conductivity and $\mathrm{L}$ is characteristic length.

Frequently, finite solids can be approximated as plates, cylinders and spheres. Thus, analytical solutions of heat conduction transients remain useful for engineering calculations and education. Early "surface" transients driven by convection are modeled using the semi-infinite solid solution along the surface assumed to be flat (Glicksman and Lienhard 2016, Lienhard and Lienhard 2017, Incropera and De Witt 1990, Kakac and Yener 1993, Arpaci 1966):

$$
\begin{gathered}
\frac{\theta(x, t)}{\theta_{i}}=\operatorname{erf} \eta+e^{2 \beta \eta+\beta^{2}} \operatorname{erfc}(\eta+\beta) \\
\eta=\frac{x_{1}}{2 \sqrt{\alpha t}}=\frac{\xi}{2 \sqrt{F o}}
\end{gathered}
$$




$$
\beta=\frac{h \sqrt{\alpha t}}{k}=B i \sqrt{F O}
$$

where $\mathrm{x} 1=\mathrm{L}-\mathrm{x}$ is distance from the surface. Fourier number is defined as:

$$
F o=\frac{\alpha t}{L^{2}}
$$

where $t$ is time, $\alpha$ is thermal diffusivity. $L$ is $1 / 2$ plate thickness. For cylinders and spheres, $L$ is replaced by $R$.

Fourier series solution for finite solids can be presented in the following concise form (Glicksman and Lienhard 2016):

$$
\frac{T-T_{f}}{T_{i}-T_{f}}=\frac{\theta}{\theta_{i}}=\sum_{n=1}^{\infty} A_{n} f_{n} e^{-\lambda_{n}^{2} F o}
$$

Coefficients $A_{n}$, functions $f_{n}$ and eigenvalue equations for $\lambda_{n}$ are given in Table 1 . where $\xi=x / L$ or $\xi=r / R$.

Fourier series in Eq. (2) converges rapidly, because of the negative argument of the exponential term, $e^{-\lambda_{n}{ }^{2} F o}$. One term is sufficient for $F o>0.2$ (Glicksman and Lienhard 2016, Lienhard and Lienhard 2017). The key obstacle preventing frequent practical use of Eq. (2), are eigenvalue equations, which are transcendental. $\lambda_{n}$ appears two times in each equation, which has to be solved numerically. For practical engineering calculations, most textbooks provide:

i. Tabulated values of $\lambda_{1}$ and $A_{1}$ (Glicksman and Lienhard 2016, Incropera and De Witt. 1990). Carslaw and Jaeger (1959) provide the first six eigenvalues.

ii. Transient response charts, e.g. Heisler charts (Heisler 1947) for $0.2<$ Fo $<700$ [3,4] or charts for the range $0<F o<1.5$ (Lienhard and Lienhard 2017, Arpaci 1966).

Schneider's monograph (Schneider 1963) contains numerous high-resolution charts, extending the range of Fourier numbers to $F o=0.001$. More recently (Ostrogorsky 2009), we provided a set of correlations for $\lambda_{1}$ and $A_{1}$ as a function of Bi. These are applicable for Fo $>0.2$ and all $\mathrm{Bi}$, with $<1 \%$ error (Glicksman and Lienhard. 2016, Ostrogorsky 2009).

\begin{tabular}{|c|c|c|c|}
\hline Plate & $2 \frac{\sin \lambda_{\mathrm{n}}}{\lambda_{\mathrm{n}}+\sin \lambda_{\mathrm{n}} \cos \lambda_{\mathrm{n}}}$ & $\begin{array}{c}\text { Function } f_{\mathrm{n}} \\
\left(\text { for } \xi=1, f_{\mathrm{n}}=1\right)\end{array}$ & Eigenvalue equation \\
\hline Cylinder & $2 \frac{B i}{\left(\lambda_{\mathrm{n}}^{2}+B i^{2}\right) J_{0}\left(\lambda_{\mathrm{n}}\right)}$ & $J_{0}\left(\lambda_{\mathrm{n}} \xi\right)$ & $\lambda_{\mathrm{n}} \tan \lambda_{\mathrm{n}}=B i$ \\
\hline Sphere & $2 \frac{\sin \lambda_{\mathrm{n}}-\lambda_{\mathrm{n}} \cos \lambda_{\mathrm{n}}}{\lambda_{\mathrm{n}}-\sin \lambda_{\mathrm{n}} \cos \lambda_{\mathrm{n}}}$ & $\frac{\sin \left(\lambda_{\mathrm{n}} \xi\right)}{\lambda_{\mathrm{n}} \xi}$ & $\lambda_{\mathrm{n}} \cot \lambda_{\mathrm{n}}=1-B i$ \\
\hline
\end{tabular}

Table 1. Coefficients, functions and eigenvalue equations for Eq. (2) (Glicksman and Lienhard 2016, Lienhard and Lienhard 2017) 


\section{Correlations for eigenvalues and coefficients}

Our goal is to present a set of explicit three-term approximations of the Fourier series solution, that can be used for all Biot numbers in the time-range not covered by Eq. (1). For plates, cylinders and spheres these approximations are:

$$
\begin{aligned}
\frac{\theta}{\theta_{\text {i plate }}} & =\sum_{n=1}^{m} A_{n} \cos \left(\lambda_{n} \xi\right) e^{-\lambda_{n}^{2} F o} \\
\frac{\theta}{\theta_{i c y l}} & =\sum_{n=1}^{m} A_{n} J_{0}\left(\lambda_{n} \xi\right) e^{-\lambda_{n}^{2} F o} \\
\frac{\theta}{\theta_{i s p h}} & =\sum_{n=1}^{m} A_{n} \frac{\sin \left(\lambda_{n} \xi\right)}{\lambda_{n} \xi} e^{-\lambda_{n}^{2} F o}
\end{aligned}
$$

where $m=1,2$ or 3 . The eigenvalues $\lambda_{1}, \lambda_{2}$, and $\lambda_{3}$ and Fourier's coefficients, $A_{1}, A_{2}$ and $A_{3}$ are listed in Table 2. The one-term $(m=1)$ correlations were reported previously (Glicksman and Lienhard 2016, Ostrogorsky 2009). Note that for $\xi=0, \cos (0)=1, \quad J_{0}(0)=1$ and $\lim _{x \rightarrow 0}[\sin (0) / 0]=1$.

\begin{tabular}{|c|c|c|}
\hline \multirow{4}{*}{ Plate } & Eigenvalues & Coefficient \\
\cline { 2 - 4 } & $\lambda_{1}=\frac{\pi}{2}\left(1+2.62 / B i^{1.07}\right)^{-0.468}$ & $A_{1}=1+0.273\left(1+2.42 / B i^{1.5}\right)^{-2 / 3}$ \\
\cline { 2 - 4 } Cylinder & $\lambda_{3}=2 \pi+\frac{\pi}{2}\left(1+6.733 / B i^{1.2}\right)^{-0.833}$ & $A_{2}=-0.4244\left[1+3.5 / B i^{1.45}\right]^{-1 / 1.45}$ \\
\cline { 2 - 4 } & $\lambda_{2}=3.8317+1.668\left(1+9.5 / B i^{1.25}\right)^{-1 / 1.25}$ & $A_{3}=0.2546\left[1+15.3 / B i^{1.63}\right]^{-1 / 1.63}$ \\
\cline { 2 - 4 } & $\lambda_{3}=7.016+1.628\left(1+15.85 / B i^{1.2}\right)^{-0.833}$ & $A_{1}=-1.065\left(1+7 / B i^{1.57}\right)^{-0.637}$ \\
\hline \multirow{5}{*}{ Sphere } & $\lambda_{2}=4.5+1.77\left(1+15 / B i^{1.35}\right)^{-0.7407}$ & $A_{3}=0.85\left(1+24 / B i^{1.7}\right)^{-0.588}$ \\
\cline { 2 - 4 } & $\lambda_{3}=7.72+1.7\left(1+16 / B i^{1.2}\right)^{-0.833}$ & $A_{1}=1+\left(1+8.87 / B i^{1.76}\right)^{-0.568}$ \\
\cline { 2 - 4 } & $\lambda_{3}=-2\left(1+11.22 / B i^{1.62}\right)^{-0.6173}$ \\
\hline
\end{tabular}

Table 2. Explicate correlations for eigenvalues and coefficients

\section{Exact solutions and correlations}

Figures 1, 2 and 3 demonstrate the precision of eigenvalue correlations given in Table 2 . Numerically calculated eigenvalues, labeled num[1], num[2] and num[3], are considered to be 
exact with five significant digits. lamda_1, lamda_2 and lamda_3 are calculated using the correlations given in Table 2. The correlation error:

$$
\% \text { error }=\frac{\text { num }[n]-l a m d a_{-} n}{n u m[n]} \times 100
$$

is below $0.6 \%$, see Figs. $1 \mathrm{~b}, 2 \mathrm{~b}$ and $3 \mathrm{~b}$.

a) PLATE: 1 st, $2^{\text {nd }}$ and $3 r d$ eigenvalue

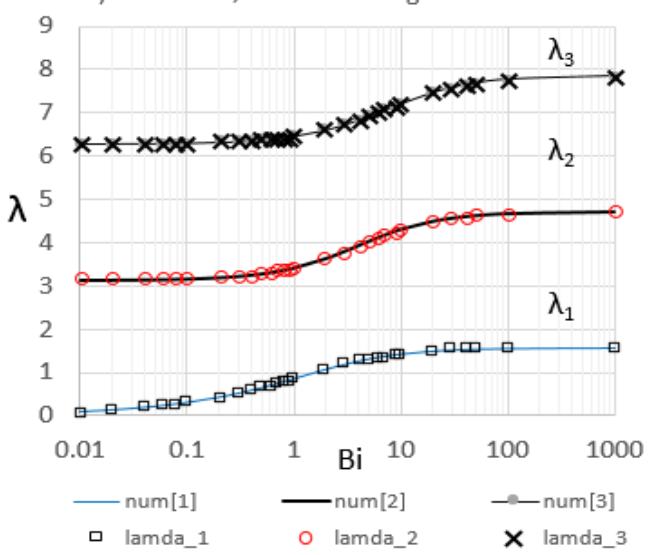

b) PLATE: \% error in eigenvalues

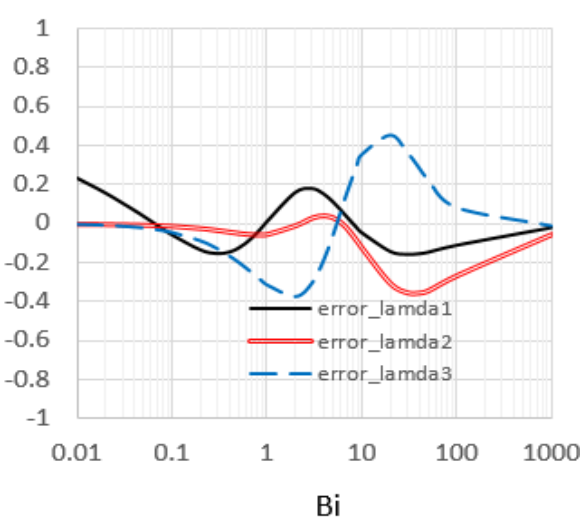

Fig. 1. a) num[1], num[2] and [3] are numerical solutions of the eigenvalue equation for plates, Table 1. lamda_1, lamda_2 and lamda_3 were calculated using correlations in Table 2; b) Error $<0.43 \%$

a) CYLINDER: 1st, 2nd and $3^{\text {rd }}$ eigenvalue

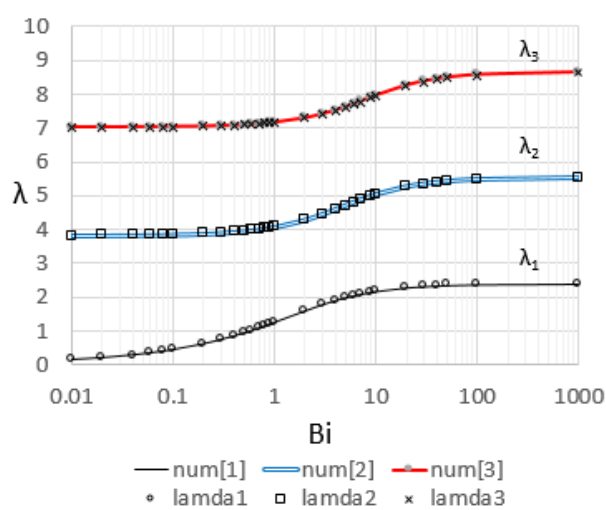

b) CYLINDER: \% error in eigenvalues

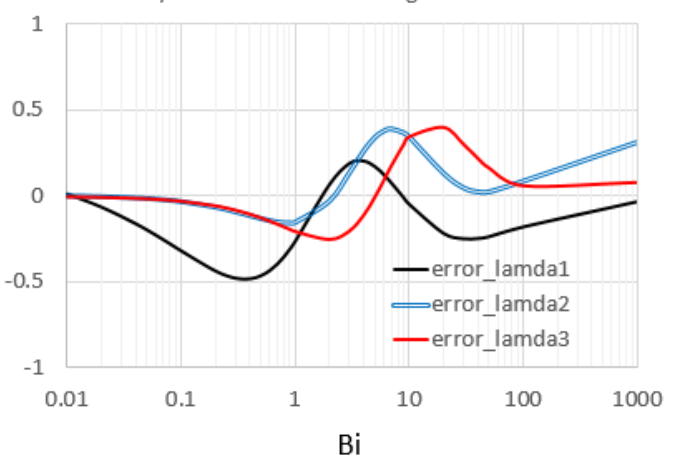

Fig. 2. a) num[1], num[2] and [3] are numerical solutions of the eigenvalue equation for cylinders, Table 1. lamda_1, lamda_2 and lamda_3 were calculated using the correlations in Table 2; b) Error $<0.5 \%$ 


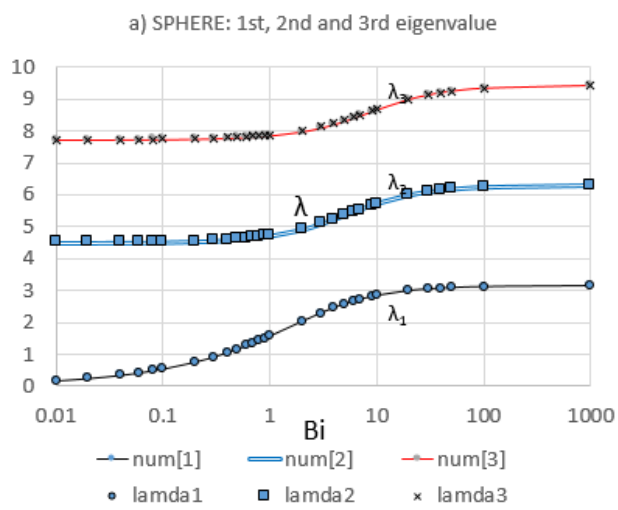

b) SPHERE: \% error eigenvalues

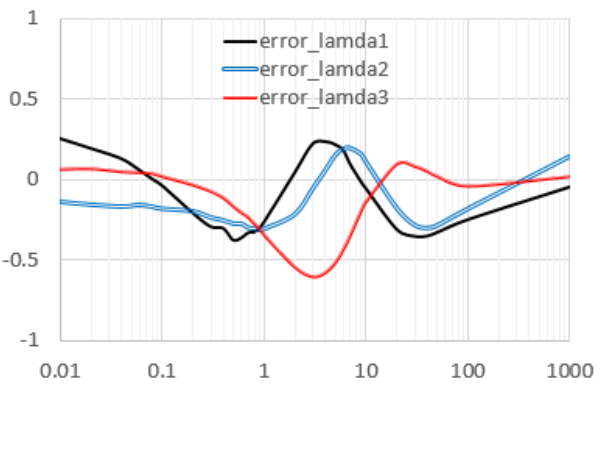

Fig. 3. a) num[1], num[2] and [3] are numerical solutions of the eigenvalue equation for spheres, Table 1. lamda_1, lamda_2 and lamda_3 are correlations given in Table 2. b) Error < $0.6 \%$

In Figs. 4 to 8, Eqs. (3), (4) and (5) with $m=1$ to 3, are compared to the exact solutions (10term Fourier series, full lines labeled Exact). Dashed blue line with circles represent the semiinfinite solid model, Eq. (1). Errors in dimensionless temperature are:

$$
\% \text { error }=\frac{\theta_{\text {exact }}-\theta_{\text {approx. }}}{\theta_{i}} \times 100
$$

Plates having $2 L$ thickness "behave" as semi-infinite, as long as the transient penetration depth $\delta$ is less than $L$. The penetration depth is:

$$
\delta=3.65 \sqrt{\alpha t}=3.65 L \sqrt{F O}
$$

setting $\delta=L$ in Eq. (8) gives Fo=0.075. Therefore, the semi-infinite solid solution is expected to be virtually exact for $0<\mathrm{Fo} \leq 0.075$. Accordingly, for $\mathrm{Fo}=0.075$ and $\mathrm{Bi}=10$, Eq. (1) gives $<0.55 \%$ error. The proposed Eq. (3) with two-terms $(m=2$, labeled T1+T2) is as precise with $<0.53 \%$ error, see Fig. 4b. In contrast, one-term Fourier solution (T1) gives $<10.3 \%$ error.

a) PLATE: $T(x / L) ; F o=0.075, \mathrm{Bi}=10$

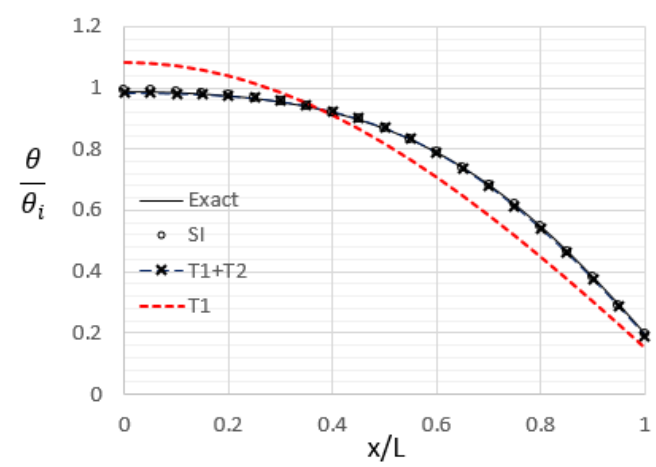

b) PLATE: \% error

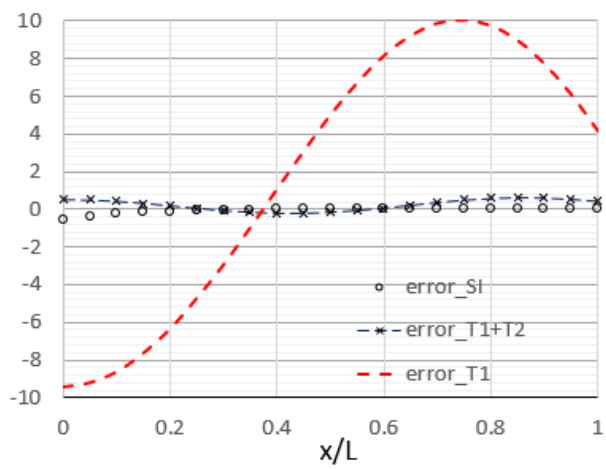

Fig. 4. $\mathrm{Fo}=0.075, \mathrm{Bi}=10$. a) Dimesionless temperature profile; b) \% error. SI is Eq. (1) 
The semi-infinite solid model can be applied to cylinders and spheres for a short period of time, while transient penetration depth $\delta$ is small compared to $R(\delta<<R)$, In Fig. 5a, Fo=0.055 was chosen because at that time $\delta=R^{1}$. For $\delta=R$, Eq. (4) with three terms $(m=2)$ is exceptionally precise, giving $<0.25 \%$ error. However, the semi-infinite solid model requires $\delta$ $<<R$ and thus yields $6.49 \%$ error, see Fig. $5 b$.

a) CYLINDER: T(r/R); Fo=0.055, $\mathrm{Bi}=10$

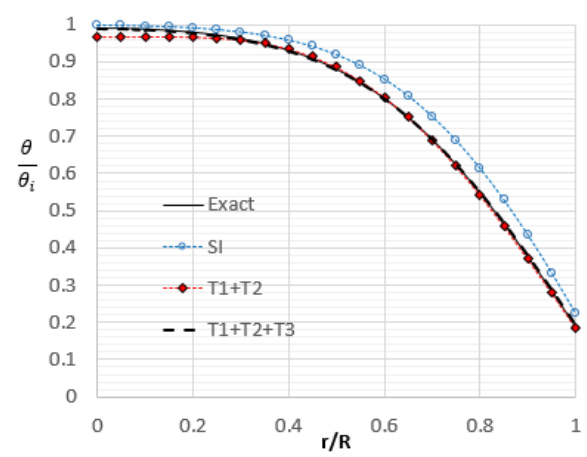

b) CYLINDER: \% error; $\mathrm{Fo}=0.055, \mathrm{Bi}=10$

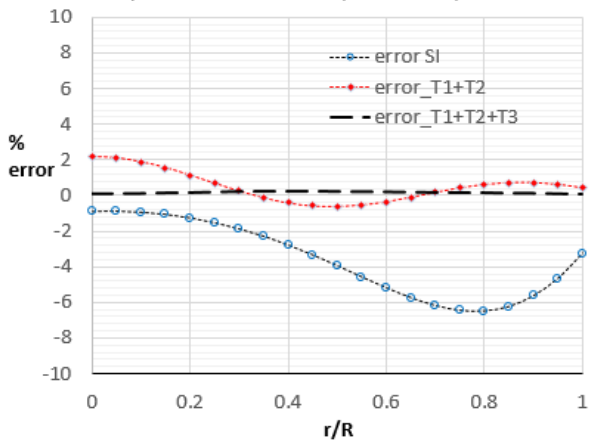

Fig. 5. Cylinder, Fo=0.055, $\mathrm{Bi}=10$. a) Dimesionless temperature profile; b) \% error. SI is Eq. (1) $\mathrm{T} 1+\mathrm{T} 2$ and $\mathrm{T} 1+\mathrm{T} 2+\mathrm{T} 3$ are Eq. (4) with $m=2$ and $m=3$ respectively

In Fig. $6 \mathrm{a}, \delta \approx 1 / 2 R$ corresponds to Fourier number $F_{O}=0.02$. As a result, error in Eq. (1) is reduced to $<3.22 \%$, while Eq. (4) with three-terms (T1+T2+T3), gives $<3.9 \%$ error, Fig. $6 \mathrm{~b}$.
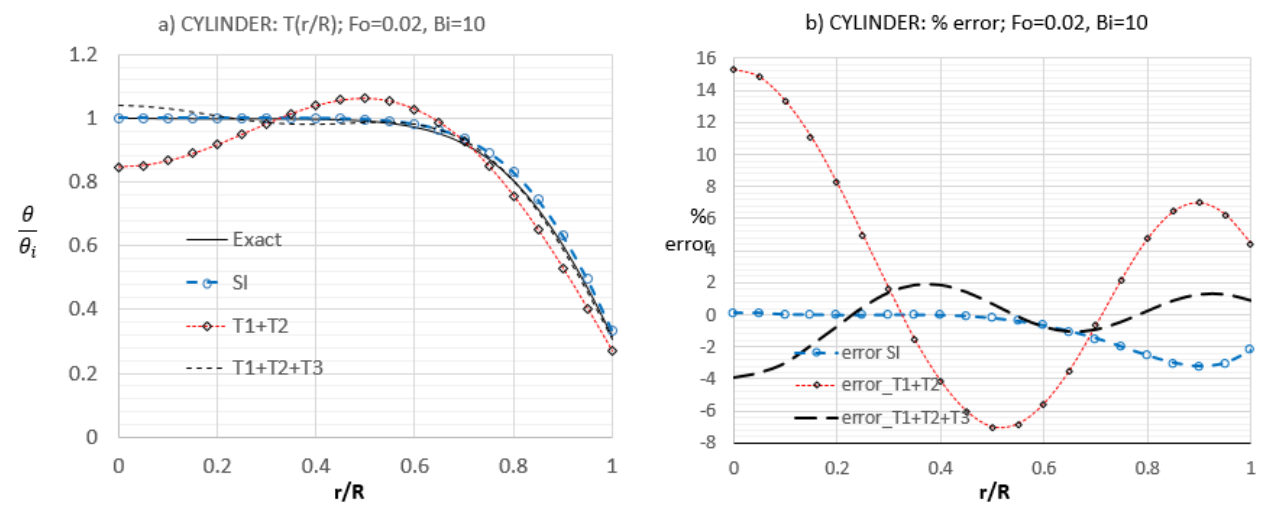

Fig. 6. Cylinder, $\mathrm{Fo}=0.02, \mathrm{Bi}=10$. a) Dimesionless temperature profile; b) \% error. $\mathrm{SI}$ is Eq. (1) $\mathrm{T} 1+\mathrm{T} 2$ and $\mathrm{T} 1+\mathrm{T} 2+\mathrm{T} 3$ are Eq. (4) with $m=2$ and $m=3$ respectively

For spheres, $\delta \approx R$ at Fo=0.045 see Fig. 7a. At that time, Eq. (1) gives $<11.5 \%$ error, while Eq. (5) with two- and three-terms gives $<6 \%$ and $<0.36 \%$, respectively, see Fig. $7 \mathrm{~b}$.

\footnotetext{
${ }^{1}$ For cylinders, at $\mathrm{Fo}=0.055$ and $r / R=0, \theta / \theta_{i}=0.99$
} 

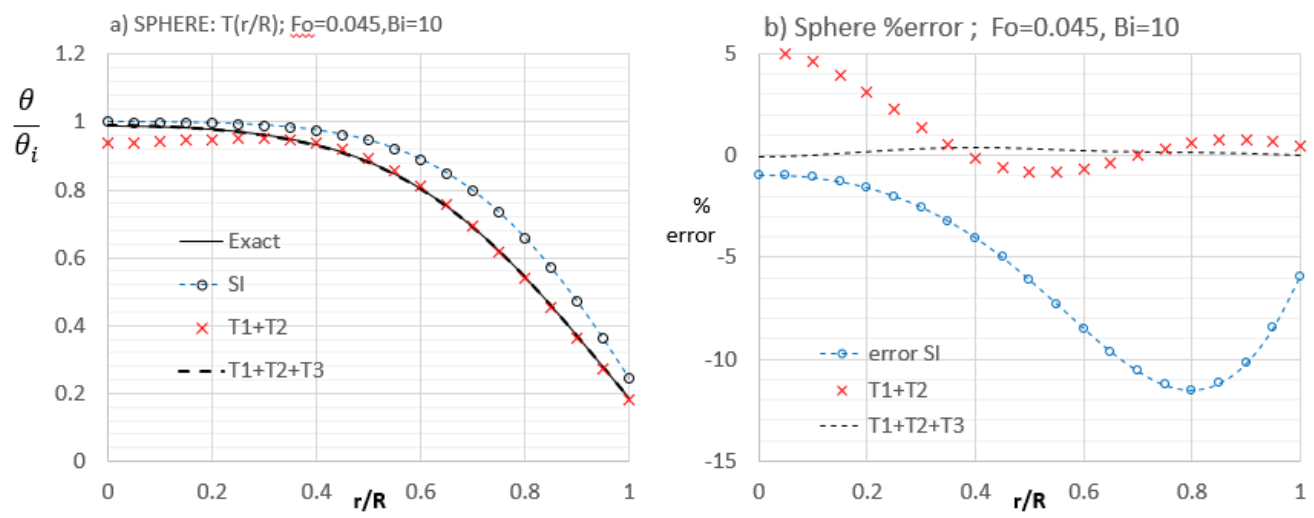

Fig. 7. Sphere, $\mathrm{Fo}=0.045, \mathrm{Bi}=10$. a) Dimesionless temperature profile; b) $\%$ error in SI model, Eq. (1). T1+T2 and T1+T2+T3 are Eq. (5) with $m=2$ and $m=3$ respectively

In Fig. 8. to make the precision of Eqs. (1) and (5) more even, Fourier number is set to $\mathrm{Fo}=0.02$. As a result, Eq. (1) gives $<6.5 \%$ error while Eq. (5) with three-terms (T1+T2+T3, $m=3$ ), gives $7.4 \%$ error at $r / R=1$. Error is $<2 \%$ for $0.25<r / R<1$ (see Fig. $8 b$ ).

a) SPHERE: $T(r / R) ; F o=0.02, \mathrm{Bi}=10$

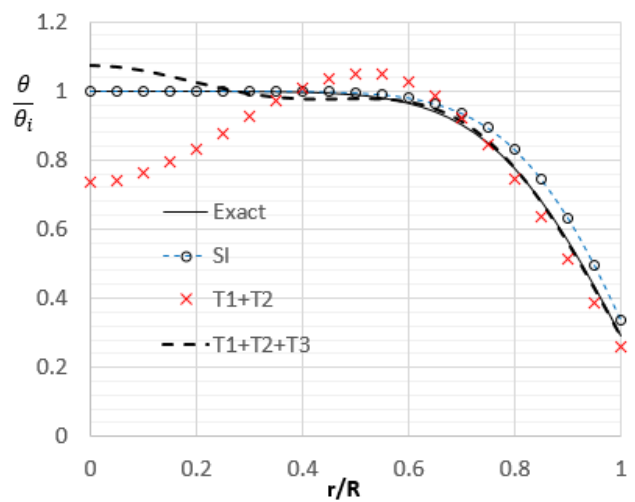

b) Sphere \%error ; $\mathrm{Fo}=0.02, \mathrm{Bi}=10$

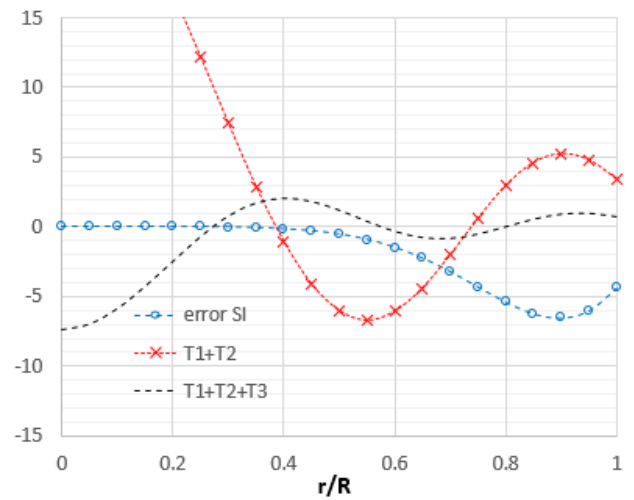

Fig. 8. Sphere, $\mathrm{Fo}=0.02, \mathrm{Bi}=10$. a) Dimesionless temperature profile; b) \% error. SI is Eq.(1)

\section{Conclusions}

The transcendental eigenvalue equations require numerical iterations, and thus prevent practical use of Fourier series solutions for heat conduction transients driven by convection. To avoid iterations or interpolating tableted values, explicit correlations were derived for:

- $\quad \lambda_{1}, \lambda_{2}$ and $\lambda_{3}$, and

- $\quad \mathrm{A}_{1}, \mathrm{~A}_{2}$ and $\mathrm{A}_{3}$, 
see Table 2. The correlations yield: $<0.42 \%$ error for plates, $<0.5 \%$ for cylinders and $<0.6 \%$ for spheres (see Figs. 1-3).

Figure 9 shows the errors in the semi-infinite solid solution, Eq. (1) and proposed Eqs. (4) and (5). Considering the $+/-10 \%$ typical uncertainty in Biot numbers, $<2 \%$ error appears appropriate for practical calculations. Therefore, the useful time-range of the semi-infinite solid solution should not be starched beyond $F_{O} \approx 0.005$ for cylinders and $F_{O} \approx 0.01$ for spheres. In conclusion, based on the errors shown in Fig. 9, the following is recommended:

- Semi-infinite solid solution, Eq. (1), should be used in the range $0<F_{O}<0.01$ for cylinders and $0<F O<0.005$ for spheres.

- Two-term approximation should be used in the range $0.06<\mathrm{Fo}<\infty$;

- $\quad$ Three-term approximation should be used in the range $0.03<\mathrm{Fo}<\infty$.

Note: The recently proposed "extended" semi-infinite solid model (Ostrogorski and Mikic, 2017), dotted line with + in Fig. 9 is sufficiently precise in the $0<F o \leq 0.06$ range. This "extended" semi-infinite solid solution combines Eq. (1) with effective or "extended" Fourier number, $\mathrm{Fo}^{+}$:

$$
\begin{gathered}
\mathrm{FO}^{+}=\frac{F O}{(1-C \sqrt{F O})^{2}} \\
\eta=\frac{\xi}{2 \sqrt{F O^{+}}} ; \quad \beta=B i \sqrt{F_{O^{+}}} \\
\frac{\theta(r, t)}{\theta_{i}}=\operatorname{erf} \eta+e^{2 \beta \eta+\beta^{2}} \operatorname{erfc}(\eta+\beta)
\end{gathered}
$$

where $\mathrm{C}=1 / 2$ for cylinders and $\mathrm{C}=1$ for spheres. $F O$ is standard Fourier number based on radius $\mathrm{R}$,

$$
F o=\frac{\alpha t}{R^{2}}
$$

Dotted line with + in Fig. 9 was calculated using Eq. (9). 


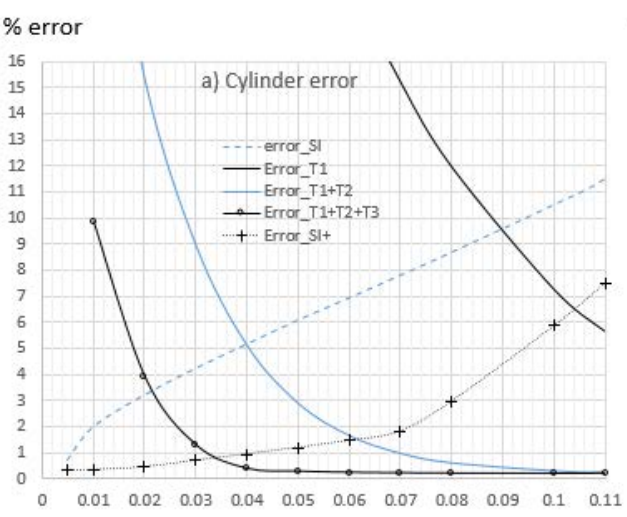

Fo

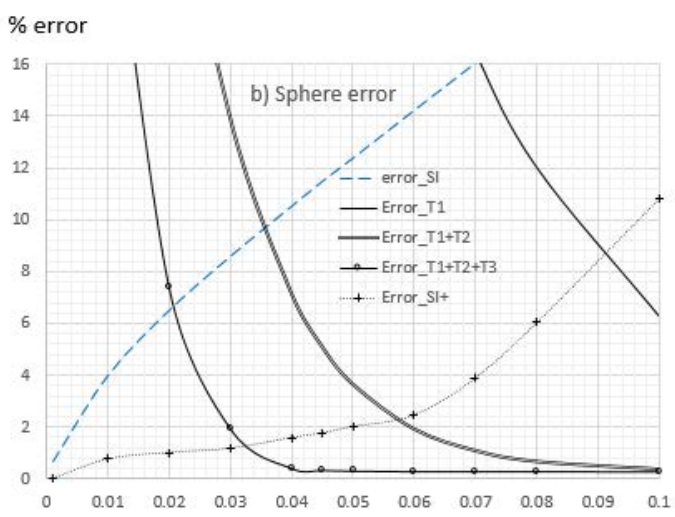

Fo

Fig. 9. Error in semi-infinite solid (SI) solution, Eq. (1) and a) Eq. (4) for cylinders and b) Eq.

(5) for spheres. T1, T1+T2, and T1+T2+T3 correspond to one- two- and three-term approximations. Error $\mathrm{SI}^{+}$is error in Eq. (9) with a) $C=1 / 2$ for cylinders, and b) $C=1$ for spheres

Acknowledgment The author would like to thank Professor John Lienhard of Massachusetts Institute of Technology, for valuable suggestions and interest in this project.

\section{References}

Arpaci V (1966). Conduction Heat Transfer, Addison-Wesley, Reading, MA.

Carslaw and Jaeger (1959). Conduction of Heat in Solids, Oxford Univ. Press, 2nd ed.

Glicksman LR, and Lienhard VJH (2016). Modeling and Approximation in Heat Transfer, Cambridge University Press.

Heisler MP (1947). Temperature Charts for Induction and Constant-Temperature Heating, Trans. ASME, 69, 227-236.

Incropera FP and De Witt DP (1990). Introduction to Heat Transfer, 2nd ed., Willey, New York.

Lienhard JH IV and Lienhard JH (2017). A Heat Transfer Textbook, 4th ed. Phlogiston Press, Cambridge MA. http://ahtt.mit.edu

Kakac S and Yener Y (1993). Heat Conduction, 3rd ed., Taylor \& Fancis, London.

Ostrogorsky AG (2009). Simple Explicit Equations for Transient Heat Conduction in Finite Solids, ASME J. Heat Transfer 131 011303-1.

Ostrogorsky AG and Mikic BB (2017). Semi-infinite solid solution extended to cylindrical and spherical solids, using time dependent participating volume-to-surface ratio and erfc solutions", ASME J. Heat Transfer, submitted.

Schneider PJ (1963). Temperature Response Charts, John Wiley and Sons, Inc. New York and London. 